\title{
A Note on Risk Aversion, Prudence and Portfolio Insurance
}

\author{
Philippe Bertrand ${ }^{\mathrm{a}}$ and Jean-Luc Prigent ${ }^{\mathrm{b}}$ \\ ${ }^{a}$ GREQAM, University Aix-Marseille II \& Euromed Management, Marseille, France. \\ E-mail: philippe.bertrand@univmed.fr \\ ${ }^{\mathrm{b}}$ THEMA, University Cergy-Pontoise, 33 Bd du Port, Cergy-Pontoise 95011, France. \\ E-mail: jean-luc.prigent@u-cergy.fr
}

This paper examines some properties of portfolio insurance that are linked to the risk aversion and the prudence of the investor. We provide explicit conditions to measure portfolio sensitivity to downside risk. We also characterize the degree of portfolio insurance by means of the ratio of absolute prudence to absolute risk aversion.

The Geneva Risk and Insurance Review (2010) 35, 81-92. doi:10.1057/grir.2009.8; published online 20 April 2010

Keywords: portfolio insurance; prudence; risk aversion

Jel classification: D81; G11

\section{Introduction}

Since the seminal results of Pratt (1964) and Arrow (1965), it has been recognized that the ratio $A(x)=-u^{\prime \prime}(x) / u^{\prime}(x)$ (the so-called Absolute Risk Aversion) is an appropriate measure of degree of risk aversion. Indeed, an individual with utility function $u_{1}$ is more risk-averse than an individual with utility function $u_{2}$ (i.e. $u_{1}$ is a concave transformation of $u_{2}$ ) if $A_{1} \geqslant A_{2}$. In that case, risk premium $\pi_{1}$ is higher than risk premium $\pi_{2}$. The literature on precautionary saving (see Leland, 1968) shows that precautionary saving is linked to the convexity of marginal utility $u^{\prime}$, or equivalently to the concavity of $-u^{\prime}$. Assuming the existence of the third derivative $u^{\prime \prime \prime}$, this is equivalent to the positivity of $u^{\prime \prime \prime}$. By analogy with risk aversion, Kimball (1990) introduces Absolute Risk Prudence $P(x)=-u^{\prime \prime \prime}(x) / u^{\prime \prime}(x)$ to measure the intensity of the precautionary saving motive. The idea is to substitute $-u^{\prime}$ for $u$. An agent with utility function $u_{1}$ is more prudent than an agent with utility function $u_{2}$ if $P_{1} \geqslant P_{2}$. Precautionary premium $\psi_{1}$ is higher than precautionary premium $\psi_{2}$. The notion of prudence has also been introduced to examine the demand for insurance (see Eeckhoudt and Kimball, 1992). Additionally, Eeckhoudt and Gollier (2005) have analysed the link between prudence and optimal prevention. 
In this note, we examine the joint influence of risk aversion and prudence on portfolio insurance design. The portfolio optimization theory usually considers that investors maximize the expected utility of portfolio values $V_{T}$ at maturity $T$. These values may be payoff functions $h$ of a given reference portfolio $S_{T}$ (also called the benchmark), usually a financial index such as the $S \& P 500$. For example, one of the standard portfolio insurance techniques is Option Based Portfolio Insurance introduced by Leland and Rubinstein (1976), which consists of a portfolio invested in a risky asset $S$ covered by a listed put written on it. More generally, portfolio insurance has been examined in the partial equilibrium framework by Leland (1980) and by Brennan and Solanki (1981).

We provide new insights on the properties of optimal payoff $h$. Let us recall, as mentioned by Leland (1980), that convexity of payoff is linked to portfolio insurance. First, we give explicit conditions based on the investor's prudence to analyse the sensitivity of portfolio payoff $h$ to downside risk, as defined by Menezes et al. (1980). Second, we show how the investor must structure her portfolio, according to both her risk aversion and prudence. More precisely, we prove that an individual with utility function $u_{1}$ has an optimal payoff $h_{1}$ more convex (resp. more concave) than the optimal payoff $h_{2}$ of an individual with utility function $u_{2}$ if $P_{1} / A_{1} \geqslant P_{2} / A_{2}$ (resp. $P_{1} / A_{1} \leqslant P_{2} / A_{2}$ ).

The next section introduces the model and presents the results. The section after that provides concluding remarks. All proofs are in the appendix.

\section{The model and our results}

In the standard optimal insurance literature, the optimal design of insurance contracts is mainly based on the determination of assumptions, under which a straight deductible or coinsurance are optimal. For example, Raviv (1979) proves that, for a risk-averse insurer, increasing and convex administrative costs imply that the optimal insurance policy displays coinsurance above a deductible. The insurance contract is usually modelled by a couple $[I(), P$.$] ,$ where $I($.$) corresponds to the indemnity paid by the insurance company with$ additional administrative costs $c(I)$ and $P$ is the premium paid by the insured client. When the client faces a loss $x$, she receives $I(x)$ from the company (usually, we assume: $0 \leqslant I(x) \leqslant x) .{ }^{1}$ The contract $[I, P]$ is optimal if it maximizes the client's expected utility of final wealth, while allowing the company to keep at least the same utility level. Denote respectively by $W_{0}$ and $\tilde{W}_{0}$, the initial wealths of the client and the insurance company, and by $U$ and $\tilde{U}$ their utility

\footnotetext{
${ }^{1}$ See Gollier (1987) and Breuer (2006) when this condition is not assumed.
} 
functions. Let $F$ be the cumulative distribution function of the potential loss. Then, the usual optimal contract $[I, P]$ is the solution of the following problem:

subject to:

$$
\max _{[I, P]} \int_{0}^{x_{\max }} U\left[W_{0}-x+I(x)-P\right] d F(x),
$$

$$
\int_{0}^{x_{\max }} \tilde{U}\left[\tilde{W}_{0}+P-I(x)-c(I(x))\right] d F(x) \geqslant \tilde{U}\left[\tilde{W}_{0}\right] .
$$

The determination of the optimal solution allows to analyse the effect of risk aversion on the optimal deductible and on the coinsurance rate.

In the finance literature, the optimal design of financial portfolio is studied in a different framework. We search for an optimal payoff schedule $h($.$) defined as a function of a given financial asset return (usually, one of$ the main financial indices). Therefore, we do not focus only on losses but also on potential gains. ${ }^{2}$ Additionally, the standard model of insurance assumes a constant loading, while the financial model supposes that derivative instruments can be used to hedge the portfolio. Finally, the constraint corresponds to a standard budget condition since only one agent is concerned: the investor.

The calculation of the optimal solution allows to examine the effect of risk aversion on the convexity/concavity of the optimal portfolio payoff.

\section{Optimal payoff as a function of a reference portfolio}

In this section, we recall the results of Leland (1980) and of Brennan and Solanki (1981). Assume that the investor maximizes the expected utility of her terminal wealth $V_{T}$ under historical probability $\mathbb{P}$. Portfolio value $V_{T}$ is assumed to be a function $h$ of risky asset value $S_{T}$. As usual, the utility $U$ of the investor is taken to be increasing, concave and twice differentiable. Under the standard condition of no-arbitrage, asset prices are calculated under a riskneutral probability $\mathbb{Q}$. Denote by $(d \mathbb{Q}) /(d \mathbb{P})$ the Radon-Nikodym derivative of

${ }^{2}$ For the optimal insurance design, the payoff $h(X)$ is given

$$
h(X)=W_{0}-X+I(X)-P,
$$

where $X$ denotes the potential loss. The variable of interest is mainly $I(X)$.

For the optimal portfolio design, $X$ denotes the financial index return, with potential gains and losses. 
$\mathbb{Q}$ with respect to historical probability $\mathbb{P}$. Denote by $N_{T}$ the discount factor and by $M_{T}$ the product $N_{T}(d \mathbb{Q}) /(d \mathbb{P})$.

Due to the no-arbitrage condition, the budget constraint corresponds to the following relation:

$$
V_{0}=\mathbb{E}_{\mathbb{Q}}\left[h\left(S_{T}\right) N_{T}\right]=\mathbb{E}_{\mathbb{P}}\left[h\left(S_{T}\right) M_{T}\right] .
$$

The investor's optimization problem is:

$$
\operatorname{Max}_{h} \mathbb{E}_{\mathbb{P}}\left[U\left(h\left(S_{T}\right)\right] \text { s.c. } V_{0}=\mathbb{E}_{\mathbb{P}}\left[h\left(S_{T}\right) M_{T}\right] .\right.
$$

Denote by $g$ the conditional expectation of $M_{T}$ under the $\sigma$-algebra generated by $S_{T}$. Then, the optimal payoff $h$ is given by: ${ }^{3}$

$$
h=J(\lambda g),
$$

where $J$ denotes the inverse of the marginal utility $\left(J=\left(U^{\prime}\right)^{-1}\right)$, and $\lambda$ is the scalar Lagrange multiplier such that

$$
V_{0}=\int_{\mathbb{R}^{+}} J(\lambda g(s)) g(s) \mathbb{P}_{S_{T}}(d s) .
$$

\section{Optimal payoff properties}

Monotonicity of the optimal payoff

Introduce tolerance of risk $T(h(s))$, which is equal to the inverse of absolute risk aversion:

$$
T(h(s))=-\frac{U^{\prime}(h(s))}{U^{\prime \prime}(h(s))} .
$$

Note that if a representative or "average" investor $r$ exists for the market, tolerance of risk $T_{r}(s)$ is equal to $-(g(s)) /\left(g^{\prime}(s)\right)$ since $g=U_{r}^{\prime}$. This is the reason why, in what follows, we denote $-(g(s)) /\left(g^{\prime}(s)\right)$ by $T_{r}(s)$. We assume that $g$ is twice-differentiable. ${ }^{4}$

${ }^{3}$ The results is proved by using the first-order condition with respect to $h$ of the following Lagrangian:

which leads to:

$$
L(h, \lambda)=\mathbb{E}_{\mathbb{P}}\left[U\left(h\left(S_{T}\right)\right)\right]+\lambda\left(V_{0}-\mathbb{E}_{\mathbb{P}}\left[h\left(S_{T}\right) M_{T}\right]\right),
$$

$$
U^{\prime}\left(h\left(S_{T}\right)\right)=\lambda M_{T} .
$$

${ }^{4}$ This assumption is not too restrictive, since it is verified for standard financial markets, for example Black and Scholes. 
Proposition 1 The first derivative $h^{\prime}$ of the optimal payoff is given by:

$$
h^{\prime}(s)=\frac{T(h(s))}{T_{r}(s)} .
$$

Thus, optimal payoff $h$ is increasing, if both the individual and the representative agent have positive risk tolerance (usual assumption).

\section{Sensitivity to downside risk}

Kimball (1990) introduces the notion of prudence within the expected-utility framework, which is characterized by the positivity of the third derivative of utility. He shows that prudence corresponds to a precautionary-savings motive. In Gollier (2001), an agent is prudent if the addition of an uninsurable risk to her future wealth with zero-mean increases her optimal saving. Eeckhoudt and Schlesinger (2006) define prudence "as a type of preference for disaggregation of two untoward events": on one side, a certain reduction in wealth; on the other side, a zero-mean independent noise added to the distribution of wealth. ${ }^{5}$

Menezes et al. (1980) also consider the case $U^{\prime \prime \prime}>0$, but by introducing the notion of downside risk, which is based on the "location of risk" in the probability distribution. According to Menezes et al. (1980), a probability distribution with density $f_{2}$ has more downside risk than a pdf $f_{1}$ if $f_{2}$ can be obtained from $f_{1}$ by a sequence of Mean-Variance-Preserving Transformations (MVPT). This means that $\operatorname{pdf} f_{2}$ is obtained from $\operatorname{pdf} f_{1}$ by shifting dispersion from right to left, without modifying expectation and variance.

Using a characterization of MVPT, we have:

\section{Proposition 2}

1. Payoff $h_{2}$ has more downside risk than payoff $h_{1}$ if and only if:

1.1. They have the same expectation: $E\left[h_{1}\left(S_{T}\right)\right]=E\left[h_{2}\left(S_{T}\right)\right]$.

1.2. $\int_{0}^{\infty} \int_{0}^{z}\left[\mathbb{P}\left[h_{2}\left(S_{T}\right) \leqslant y\right]-\mathbb{P}\left[h_{1}\left(S_{T}\right) \leqslant y\right]\right] d y d z=0$.

1.3. $\int_{0}^{x} \int_{0}^{z}\left[\mathbb{P}\left[h_{2}\left(S_{T}\right) \leqslant y\right]-\mathbb{P}\left[h_{1}\left(S_{T}\right) \leqslant y\right]\right] d y d z \geqslant 0$, for all $x$ in $\mathbb{R}^{+}$and $>0$ for some $x_{0}$ in $\mathbb{R}^{+}$.

2. The optimal payoff of any prudent investor has always less downside risk than any other payoff.

\footnotetext{
${ }^{5}$ Eeckhoudt and Schlesinger (2006) define prudence as "a type of natural preferences over simple lotteries", defined as couples of wealth reduction and additional noise. More precisely, an individual is said to be prudent if she prefers the lottery $A=[-k, \tilde{\varepsilon}]$ to the lottery $B=[0, \tilde{\varepsilon}-k]$, where all outcomes of the lotteries have equal probability, for all initial wealth levels and for any wealth reduction $k$ and noise $\tilde{\varepsilon}$.

In the same manner, they define temperance as follows. An individual is said to be temperate if she prefers the lottery $C=\left[\tilde{\varepsilon}_{1}, \tilde{\varepsilon}_{2}\right]$ to the lottery $D=\left[0, \tilde{\varepsilon}_{1}+\tilde{\varepsilon}_{2}\right]$, where all outcomes of the lotteries have equal probability, for all initial wealth levels and for any independent noises $\tilde{\varepsilon}_{1}$ and $\tilde{\varepsilon}_{2}$.
} 
We now examine the particular case where $h_{1}$ is a transformation of $h_{2}$, that is: $h_{1}=\Phi\left(h_{2}\right)$, where $\Phi$ is non-decreasing, bijective and differentiable.

Proposition 3 Payoff $h_{2}$ has more downside risk than payoff $h_{1}$ if and only if $\left[f_{1}(\Phi) \Phi^{\prime}-f_{1}\right]$ is a combination of MVPTs.

Ratio of prudence to risk aversion and convexity/concavity degree of the optimal payoff

The convexity/concavity of the payoff is an important point since it determines the investor's portfolio position in risky asset $S$. As mentioned by Leland (1980), the convexity of the payoff, which is linked to portfolio insurance, allows the investor to take advantage of market rises. For example, you can hold cash and buy a call option on $S$.

In what follows, we prove that the ratio $P / A$ of absolute prudence to absolute risk aversion plays a key role in explaining the origin of the convexity/concavity of the payoff. As mentioned in Gollier (2001), ratio $P / A$ allows absolute risk aversion and risk vulnerability to be analysed, in the presence of background risk. This result has been established for the standard portfolio optimization problem, where the amount invested in risky asset $S$ is proportional to $S$ (i.e. payoff $h(S)$ is linear). ${ }^{6}$ Here, we extend the use of this ratio to the general problem of optimal portfolio positioning, in particular to portfolio insurance.

The convexity/concavity is determined by the sign of the second-order derivative $h^{\prime \prime}(s)$.

Proposition 4 The second derivative of the optimal payoff is given by:

$$
h^{\prime \prime}(s)=\left[T^{\prime}(h(s))-T_{r}^{\prime}(s)\right] \times\left[\frac{T(h(s))}{T_{r}(s)^{2}}\right] .
$$

The previous result allows the convexity/concavity of the optimal payoff to be characterized.

\footnotetext{
${ }^{6}$ Absolute risk aversion $A$ is decreasing with wealth (DARA) if and only if ratio $P / A$ is higher than 1 , since $A^{\prime} / A^{2}=1-P / A$, which implies prudence. Under DARA, decreasing absolute prudence (DAP) implies risk vulnerability. DAP is equivalent to $A^{\prime \prime} \geqslant-\mathrm{A}^{\prime}[P-2 A]$. Thus, under DARA, if $P / A$ is higher than 2, then DAP implies the convexity of absolute risk aversion (see Gollier, 2001).

Using Arrow-Pratt approximations, note also that ratio $P / A$ is proportional to the ratio of premia $\psi / \pi$.
} 
Corollary 5 Optimal payoff $h$ is convex if and only if cautiousness $T^{\prime}(h(s))$ is higher than the cautiousness $T_{r}^{\prime}(s)$ of the representative agent. Furthermore, the convexity of optimal payoff $h$ is equivalent to the following inequality, which is satisfied by the ratios of absolute prudence divided by absolute risk aversion:

$$
\left(\frac{P}{A}\right) \geqslant\left(\frac{P_{r}}{A_{r}}\right) .
$$

Note that $P_{r}$ is usually positive. ${ }^{7}$ Thus, assuming that agents are strictly riskaverse $\left(A>0\right.$ and $\left.A_{r}>0\right)$, the convexity of optimal payoff $h$ implies prudence condition $P \geqslant 0$.

From relation (5), comparative convexity/concavity can be derived for optimal payoffs.

Proposition 6 Consider two agents with, respectively, utility functions $u_{1}$ and $u_{2}$. If both optimal payoffs are convex, then the optimal payoff $h_{1}$ of agent 1 is more convex than the optimal payoff $h_{2}$ of agent 2 if and only if

$$
\left(\frac{P_{1}}{A_{1}}\right) \geqslant\left(\frac{P_{2}}{A_{2}}\right) .
$$

If both optimal payoffs are concave, then the optimal payoff $h_{1}$ of agent 1 is more concave than the optimal payoff $h_{2}$ of agent 2 if and only if

$$
\left(\frac{P_{1}}{A_{1}}\right) \leqslant\left(\frac{P_{2}}{A_{2}}\right) .
$$

${ }^{7}$ For instance, for the Black and Scholes model, as soon as the expected return of the risky asset is higher than the riskless asset.

${ }^{8}$ Further properties of the portfolio payoff could also be related to the notion of temperance. As shown in Eeckhoudt et al. (1995), risk aversion, prudence and temperance best characterize attitude towards risk. However, temperance is based on $U^{\prime \prime \prime \prime}$, and appears in the term $h^{\prime \prime \prime}$, which is a rather involved formula. Nevertheless, temperance has an efffect on the monotonicity of the ratio $P / A:(P / A)^{\prime}$ is positive if and only if

$$
U^{\prime \prime \prime \prime}>\frac{U^{\prime \prime \prime}}{A}\left[1-2 \frac{P}{A}\right]
$$

Thus, under risk aversion and prudence, and if the ratio $P / A$ is increasing, temperance (i.e. $U^{\prime \prime \prime}<0$ ) will be verified only if $P / A>1 / 2$ (for example, for DARA, since in that case, $P / A>1)$. 
Note that, if both agents are risk-averse and prudent or are risk-averse and not prudent, conditions (7) and (8) are respectively equivalent to:

$$
\left(\frac{A_{1}}{P_{1}}\right) \leqslant\left(\frac{A_{2}}{P_{2}}\right) \text { and }\left(\frac{A_{1}}{P_{1}}\right) \geqslant\left(\frac{A_{2}}{P_{2}}\right) .
$$

Recall that payoff $h_{1}$ is more convex than payoff $h_{2}$ if and only if there exists a convex function $\Phi$ such that $h_{1}=\Phi\left(h_{2}\right)$. Since the convexity of the payoff is linked to portfolio insurance (see Leland, 1980), condition (7) proves that ratio $(P / A)$ measures the degree of portfolio insurance.

Note that the equivalence between the property " $h_{1}$ more convex than $h_{2}$ " and the inequality $\left(P_{1} / A_{1}\right) \geqslant\left(P_{2} / A_{2}\right)$ is quite similar to the equivalence " $u_{1}$ is more prudent than $u_{2}$ " and $P_{1} \geqslant P_{2}$.

\section{Conclusion}

The notion of absolute risk aversion has been extensively used to describe attitude towards risk. More recently, the concept of prudence has permitted the analysis of precautionary saving. This note highlights the relationships between, on the one hand, risk aversion and prudence and, on the other hand, optimal portfolio payoff. For instance, for a prudent investor, the optimal payoff always has less downside risk than any other. We also prove that the ratio of absolute prudence to absolute risk aversion determines the optimal portfolio profile. As a by-product, we show that this ratio allows the demand for portfolio insurance to be measured. This property is linked to the market risk exposure, which measures the investor's propension to risk in order to benefit from financial market rises. Such results can be applied for example to the optimal asset allocation of pension funds (see Romaniuk, 2007).

\section{Acknowledgements}

We are grateful to Alan Kirman, Olivier Scaillet and Louis Eeckhoudt for discussions about an earlier version of this paper. We would like to thank the editor Pierre Picard, and two anonymous referees, whose comments and suggestions have permitted to substantially improve the quality of this paper.

\footnotetext{
${ }^{9}$ Note also that the equivalence between the property " $h_{1}$ more concave than $h_{2}$ " and the inequality $\left(A_{1} / P_{1}\right) \geqslant\left(A_{2} / P_{2}\right)$ is quite similar to the equivalence " $u_{1}$ is more risk-averse than $u_{2}$ " and $A_{1} \geqslant A_{2}$, and also to the equivalence " $u_{1}$ is more prudent than $u_{2}$ " and $P_{1} \geqslant P_{2}$.
} 


\section{References}

Arrow, K.J. (1965) Aspects of the Theory of Risk-Bearing, Helsinki: Yrj Hahnsson Foundation.

Brennan, M.J. and Solanki, R. (1981) 'Optimal portfolio insurance', Journal of Financial and Quantitative Analysis 16(3): 279-300.

Breuer, M. (2006) 'Optimal insurance contracts without the non-negativity constraint on indemnities: Revisited', The Geneva Risk and Insurance Review 31: 5-9.

Eeckhoudt, L. and Gollier, C. (2005) 'The impact of prudence on optimal prevention', Economic Theory 26: 989-994.

Eeckhoudt, L., Gollier, C. and Schneider, T. (1995) 'Risk aversion, prudence and temperance: A unified approach', Economics Letters 48: 331-336.

Eeckhoudt, L. and Kimball, M. (1992) 'Background risk, prudence, and the demand for insurance', in G. Dionne (ed.) Contribution to Insurance Economics, Boston, Dordrecht, London: Kluwer Academic Publishers.

Eeckhoudt, L. and Schlesinger, H. (2006) 'Putting risk in its proper place', American Economic Review 96: 280-289.

Gollier, C. (1987) 'The design of optimal insurance contracts without the nonnegativity constraint on claims', The Journal of Risk and Insurance 54: 314-324.

Gollier, C. (2001) The Economics of Risk and Time, Cambridge, Massachusetts: MIT Press.

Kimball, M.S. (1990) 'Precautionary saving in the small and in the large', Econometrica 58: $53-73$.

Leland, H. (1968) 'Saving and uncertainty: The precautionary demand for saving', The Quarterly Journal of Economics 82(3): 465-473.

Leland, H. (1980) 'Who should buy portfolio insurance', Journal of Finance 35: 581-594.

Leland, H.E. and Rubinstein, M. (1976) 'The evolution of portfolio insurance', in D.L. Luskin (ed.) Portfolio Insurance: A Guide to Dynamic Hedging, Chichester, England: Wiley.

Menezes, C., Geiss, C. and Tressler, J. (1980) 'Increasing downside risk', American Economic Review 70(5): 921-931.

Pratt, J.W. (1964) 'Risk aversion in the small and in the large', Econometrica 32: 122-136.

Raviv, A. (1979) 'The design of an optimal insurance policy', American Economic Review 69: 84-96.

Romaniuk, K. (2007) 'The optimal asset allocation of the main types of pension funds: A unified approach', Geneva Risk and Insurance Review 32: 113-128.

\section{About the Authors}

Philippe Bertrand holds a Ph.D. in Economics from EHESS. He is currently a Professor of Finance at University Aix-Marseille 2 and an affiliate Professor of Finance at Euromed Management. He conducts active research in portfolio management, performance evaluation and portfolio insurance. He was formerly the head of Financial Engineering at CCF Capital Management.

Jean-Luc Prigent holds a Ph.D. in Mathematics from University of Rennes 1 (France). He has also the "Habilitation" in Economics from the University of Cergy-Pontoise and the "Habilitation" in Business Administration from the University of Paris 1-Panthéon-Sorbonne. He is currently Professor in Economics and Finance at University of Cergy-Pontoise. He works on portfolio optimization, option pricing and hedging, risk management and utility theory. 


\section{Appendix}

Proof of Proposition 1 Differentiating Relation (2) with respect to $s$, we deduce:

$$
h^{\prime}(s)=\left(\frac{g^{\prime}(s)}{U^{\prime \prime}(h(s))}\right) \times \lambda .
$$

From Relation (2), the value of parameter $\lambda$ is:

$$
\lambda=\left(\frac{U^{\prime}(h(s))}{g(s)}\right) .
$$

Therefore, the first-order derivative of optimal payoff $h$ is:

$$
h^{\prime}(s)=\left(-\frac{U^{\prime}(h(s))}{U^{\prime \prime}(h(s))}\right) \times\left(-\frac{g^{\prime}(s)}{g(s)}\right) .
$$

Finally from Definition (3), the first derivative $h^{\prime}$ of the optimal payoff satisfies:

$$
h^{\prime}(s)=\frac{T(h(s))}{T_{r}(s)} .
$$

Note that Relation (A.3) proves that optimal payoff $h$ is an increasing function if and only if function $g$ is decreasing.

Proof of Proposition 2 We use results of Menezes et al. (1980), by substituting payoff $h$ for $\operatorname{pdf} f$. Function $f$ is the pdf of random variable $h\left(S_{T}\right)$. The first part of Proposition (2) is deduced from their Theorem (1). The second assertion is based on their Theorem (2): the condition " $E\left[U\left(h_{1}\left(S_{T}\right)\right)\right] \geqslant E\left[U\left(h_{2}\left(S_{T}\right)\right)\right]$ is satisfied for any increasing and concave utility function $U$ such that $U^{\prime \prime \prime}>0$ (i.e. prudence)" is equivalent to the property " $h_{2}$ has more downside risk than $h_{1}$ ". Therefore, we deduce that if $h_{1}$ is the optimal payoff of a prudent investor, then $h_{1}$ has less downside risk than any other payoff $h_{2}$.

Proof of Proposition 3 Assume that $h_{1}=\Phi\left(h_{2}\right)$, where $\Phi$ is non-decreasing, bijective and differentiable.

The pdf of random variable $h_{2}\left(S_{T}\right)$ is given by:

$$
f_{2}=\left[f_{1}(\Phi) \Phi^{\prime}\right]
$$


Thus, $h_{2}$ has more downside risk than $h_{1}$ if and only if the function $f_{2}-f_{1}=\left[f_{1}(\Phi) \Phi^{\prime}-f_{1}\right]$ is a mean-variance preserving transformation (MVPT).

Proof of Proposition 4 Differentiating relation (A.3) with respect to $s$, we deduce:

$$
h^{\prime \prime}(s)=\frac{h^{\prime}(s) T^{\prime}(h(s)) T_{r}(s)-T(h(s)) T_{r}^{\prime}(s)}{T_{r}^{2}(s)} .
$$

Substituting $(T(h(s))) /\left(T_{r}(s)\right)$ for $h^{\prime}(s)$,we have:

$$
h^{\prime \prime}(s)=\left[T^{\prime}(h(s))-T_{r}^{\prime}(s)\right] \times\left[\frac{T(h(s))}{T_{r}(s)^{2}}\right] .
$$

Proof of Corollary 5 The first derivative of tolerance $T^{\prime}$ satisfies:

$$
\begin{aligned}
T^{\prime} & =-\left(\frac{U^{\prime}}{U^{\prime \prime}}\right)^{\prime}=-\frac{\left(U^{\prime \prime}\right)^{2}-U^{\prime} U^{\prime \prime \prime}}{\left(U^{\prime \prime}\right)^{2}}, \\
& =-1+\left(-\frac{U^{\prime}}{U^{\prime \prime}}\right)\left(-\frac{U^{\prime \prime \prime}}{U^{\prime \prime}}\right),
\end{aligned}
$$

which is equivalent to

$$
T^{\prime}=-1+\left(\frac{P}{A}\right)
$$

Thus, we have:

$$
T^{\prime}(h(s)) \leqslant T_{r}^{\prime}(s) \Leftrightarrow\left(\frac{P}{A}\right)(h(s)) \leqslant\left(\frac{P_{r}}{A_{r}}\right)(s) .
$$

\section{Proof of Proposition 6}

- Assume that both functions $h_{1}$ and $h_{2}$ are convex. The comparison of degree of convexity is equivalent to the comparison of ratios $\left(h_{i}^{\prime \prime}(s)\right) /\left(h_{i}^{\prime}(s)\right)$. From Relations (4) and (5), we have:

$$
\frac{h^{\prime \prime}(s)}{h^{\prime}(s)}=\frac{T^{\prime}(h(s))-T_{r}^{\prime}(s)}{T_{r}(s)} .
$$

Thus:

$$
\frac{h_{1}^{\prime \prime}(s)}{h_{1}^{\prime}(s)} \geqslant \frac{h_{2}^{\prime \prime}(s)}{h_{2}^{\prime}(s)} \Leftarrow \Rightarrow T_{1}^{\prime}\left(h_{1}(s)\right) \geqslant T_{2}^{\prime}\left(h_{2}(s)\right),
$$


From Relation (A.4), this is equivalent to:

$$
\left(\frac{P_{1}}{A_{1}}\right) \geqslant\left(\frac{P_{2}}{A_{2}}\right) \text {. }
$$

- If both functions $h_{1}$ and $h_{2}$ are concave, then the comparison of degree of concavity is equivalent to the comparison of ratios $-\left(h_{i}^{\prime \prime}(s)\right) /\left(h_{i}^{\prime}(s)\right)$.

Since

$$
-\frac{h_{1}^{\prime \prime}(s)}{h_{1}^{\prime}(s)} \geqslant-\frac{h_{2}^{\prime \prime}(s)}{h_{2}^{\prime}(s)} \Leftarrow \Rightarrow T_{1}^{\prime}\left(h_{1}(s)\right) \leqslant T_{2}^{\prime}\left(h_{2}(s)\right),
$$

payoff $h_{1}$ is more concave than payoff $h_{2}$ if and only if

$$
\left(\frac{P_{1}}{A_{1}}\right) \leqslant\left(\frac{P_{2}}{A_{2}}\right) \text {. }
$$

